\title{
ANAESTHETIC MANAGEMENT OF PHAEOCHROMOCYTOMA OF LESSER SAC IN A PATIENT WITH RHEUMATIC HEART DISEASE USING COMBINED GENERAL AND EPIDURAL ANAESTHESIA.
}

\author{
Chanchal Singh ${ }^{1}$, Sujata Sharma ${ }^{2}$, Namita Arora $^{3}$, Mridula Pawar $^{4}$ \\ ${ }^{I} M D$, Senior Resident, Department of Anaesthesiology \& Critical Care, ${ }^{2} M D$, Associate Professor, \\ ${ }^{3}$ DA; DNB, Specialist., ${ }^{4}$ MD, Professor, Head of Department, Dr Ram Manohar Lohia Hospital, \\ New Delhi, India \& Post Graduate Institute \\ *Corresponding author : drsinghchanchal@gmail.com
}

Key Words: Epidural, General Anaesthesia, Phaeochromocytoma of lesser sac, Mitral Regurgitation

\begin{abstract}
Phaeochromocytoma is a rare catecholamine-secreting neuroendocrine tumour, accounting for less than $0.1 \%$ of hypertensive population with a high cardiovascular morbidity and mortality if left untreated. Extra-adrenal phaeochromocytomas of lesser sac are still rarer. Due to its rarity, anaesthetic experience is also limited. Surgical resection is the only curative therapy. During surgery there is a high risk of massive release of catecholamines, which can result in potentially fatal hypertensive crisis and cardiac arrhythmias. The perioperative management of phaeochromocytoma remains a challenge for the anaesthesiologist, despite the advent of new drugs and techniques, requiring intensive preoperative preparation and vigilant intraoperative and postoperative care. This case report describes the perioperative anaesthetic management in a 38year old female patient with phaeochromocytoma of lesser sac with Rheumatic heart disease. We used combined general and epidural anaesthesia for tumour removal surgery, resulting in the use of fewer drugs and a smoother perioperative anaesthetic course and post-operative analgesia was provided with morphine supplemented through the epidural catheter. This case report highlights the importance of epidural blockade for excision of phaeochromocytoma, especially in a developing country, where the latest techniques and advanced monitoring facilities are unavailable and affordability is a major issue.
\end{abstract}

Phaeochromocytoma was first described at autopsy by Fränkel in $1886^{1}$. The word "Phaeochromocytoma" was coined by Pick $^{2}$ in 1912, meaning "Dusky coloured tumor". Phaeochromocytomas and paragangliomas are chromaffin tumors that arise from neural crest cells. The term phaeochromocytoma refers to chromaffin tumors within the adrenal medulla and extra-adrenal phaeochromocytomas (paragangliomas) are chromaffin tumors found along the paravertebral sympathetic chain extending from the base of the skull, through the neck, thorax, abdomen and pelvis. Its prevalence is around $0.1 \%$ to $0.6 \%$ in patients with hypertensive symptoms. Surgical resection is the only curative therapy. The first successful operation was performed by Roux ${ }^{3}$ in 1926. Initially, surgical resection of phaeochromocytoma had a high perioperative mortality rate of 20 to $45 \%$ which dropped to 0 to $2.9 \%$ in the last 50 years ${ }^{4}$ due to development of better imaging techniques for accurate local isation of tumor, better preoperative medical management, and improvements in surgical and anaesthetic techniques.

Several anaesthetic techniques and agents have been used for anaesthetizing phaeochromocytoma patients. Most of the techniques describe combination of different drugs through induction, maintenance, termination and postoperative period to minimize blood pressure fluctuations and to prevent dysrhythmias intraoperatively ${ }^{5}$.The combination of General anaesthesia (GA) and Epidural anaesthesia (EA) may abate the hypertensive and dysrhythmic attacks during surgery resulting in a steady haemodynamic state.

\section{Case Report:}

A 38 -year-old, $45 \mathrm{~kg}$, female patient was admitted with sudden hypertensive crisis along 
with headache, vomiting, epigastric pain and generalized weakness. Her blood pressure was $180 / 106 \mathrm{mmHg}$. She was known to have subclinical hypothyroidism for the past 6 months and was on oral thyroxine $50 \mu \mathrm{g}$ once daily. She had a history of menorrhagia and rheumatic heart disease with mitral regurgitation for the past 4 years and bilateral cataract and pulmonary tuberculosis 13 years back for which she took anti-tuberculosis treatment for 10 months. Her investigations included haematological, biochemical evaluations, coagulation profile, thyroid function test, liver and kidney function tests, ultrasonography (USG), CT scan, upper gastro intestinal endoscopy, renal doppler study, 24 hours urinary catecholamines and Vanillyl mandelic acid levels. Ultrasound of abdomen reported an enlarged $(4.8 \mathrm{~cm} \times 5 \mathrm{~cm})$, necrotic para-aortic lymph node with mesenteric lymphadenopathy, splenomegaly and left ovarian cyst. Contrast Enhanced Computed Tomography (CECT) abdomen revealed a heterogeneously enhancing mass lesion in lesser sac and retropancreatic area measuring $5 \mathrm{~cm} \times 5.1 \mathrm{~cm} \times 5.4 \mathrm{~cm}$ likely to be a Leiomyosarcoma or Gastro-intestinal stromal tumor. Electrocardiogram showed left ventricular hypertrophy. 2D ECHO showed an ejection fraction of $62 \%$, moderate mitral regurgitation, tricuspid regurgitation pulmonary regurgitation and mild concentric left ventricular hypertrophy. Diagnosis of phaeochromocytoma was established by the raised levels of catecholamines and vanillyl mandelic acid (VMA $-38.36 \mathrm{mg} / \mathrm{gm}$ creatinine) in 24-h urinary collection. All other investigations were normal. The patient was prepared for 4 weeks with $\alpha$ and $\beta$ blockers (phenoxybenzamine $10 \mathrm{mg}$ and atenolol $25 \mathrm{mg}$, twice daily) prior to surgery.

\section{Anaesthetic Management:}

Alprazolam $0.5 \mathrm{mg}$ was administered orally at night before surgery. Morning doses of oral antihypertensives were given 2 hours preoperatively with a sip of water. Preoperative blood pressure was 140/70 $\mathrm{mmHg}$; heart rate was $77 / \mathrm{min} ; \mathrm{SpO}_{2}$ was $100 \%$ in room air. After securing intravenous access with $18 \mathrm{G}$ intravenous cannula, ringer lactate infusion was started. Monitors were attached for continuous monitoring of NIBP, $\mathrm{SpO}_{2}$ and ECG. Radial artery cannulation was done under local anaesthesia for continuous blood pressure monitoring. Then using $18 \mathrm{G}$ Tuohy' needle epidural catheter was inserted in T10-11 interspace and a single bolus dose of $15 \mathrm{ml}$ of $0.25 \%$ bupivacaine was administered after a test dose of plain lignocaine. Glycopyrrolate $0.2 \mathrm{mg}$, midazolam $1 \mathrm{mg}$ and fentanyl $100 \mu \mathrm{g}$ were administered intravenously as premedication. After preoxygenation for 3 minutes, patient was induced with propofol $1 \% 100 \mathrm{mg}$ and intubation with $7.0 \mathrm{~mm}$ I.D. cuffed endotracheal tube was uneventfully faciliated with vecuronium bromide $6 \mathrm{mg}$. Lidocaine $1.5 \mathrm{mg} / \mathrm{kg}$ body weight intravenously was administered 1 minute before laryngoscopy to minimize adverse cardiovascular effects of laryngoscopy and intubation. Maintenance of anaesthesia was done by positive pressure ventilation with $\mathrm{O}_{2}$ and $\mathrm{N}_{2} \mathrm{O}$ and isoflurane, along with supplemental dose of vecuronium bromide on demand. A central venous access was established through right subclavian approach after induction for continuous central venous pressure monitoring. During the excision and manipulation of tumour mass there was fluctuation of blood pressure, the maximum was $167 / 90 \mathrm{~mm}$ of $\mathrm{Hg}$ and returned to normal without further treatment (see Figure1). Neither acute hypertensive attack nor dysrhythmia was seen intraoperatively and therefore antihypertensive agent was not administered. Urine output was adequate throughout the procedure $(1.2-1.5 \mathrm{ml} / \mathrm{kg} / \mathrm{hr})$. Residual neuromuscular blockade was completely reversed with neostigmine $0.05 \mathrm{mg} / \mathrm{kg}$ and glycopyrrolate $0.01 \mathrm{mg} / \mathrm{kg}$ and the patient was extubated. Patient was kept in ICU under close supervision for the next 48 hours for continuous haemodynamic monitoring. Post operative pain relief was facilitated in the ICU via epidural Patient Controlled Analgesia pump and a mixture of $0.0625 \%$ bupivacaine and $0.05 \mathrm{mg} / \mathrm{ml}$ of morphine was administered at $2 \mathrm{ml} / \mathrm{hr}$. Patient on demand dose was $4 \mathrm{ml}$ and the lock up interval was 30 minutes. Ondansetron $4 \mathrm{mg}$ intravenously was administered 12 hourly. There were no adverse effects like respiratory depression, pruritus, nausea and vomiting. The patient developed hypertension, for which a nitroglycerine infusion was started and titrated according to the blood pressure. On subsequent post operative days, patient's blood pressure gradually came to near normal, Nitroglycerine infusion was discontinued and patient was shifted to the ward after removal of epidural catheter on the second day and for further analgesia tramadol $100 \mathrm{mg}$ was administered intravenously. The patient was discharged home on the 9th postoperative day with oral atenolol 
$25 \mathrm{mg}$ and hydrocortisone tablet $10 \mathrm{mg}$ in the morning and $5 \mathrm{mg}$ in the night. Histological report confirmed the tumour as phaeochromocytoma.

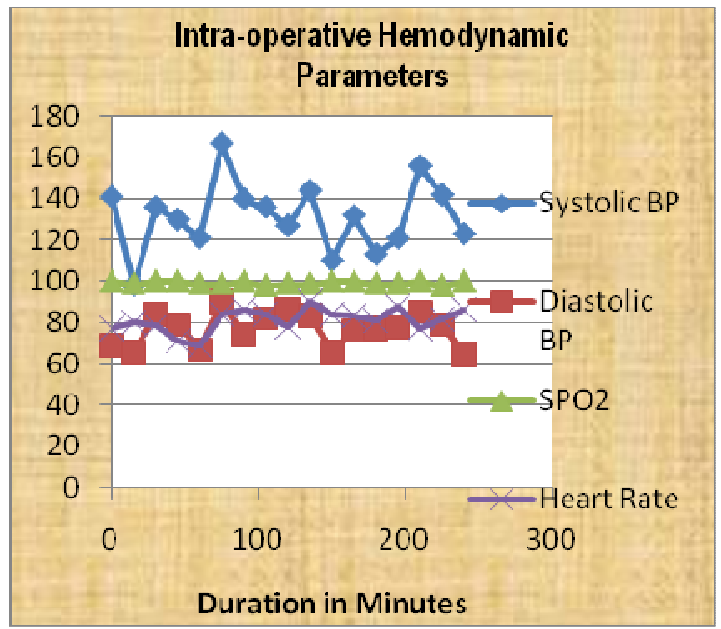

Figure - 1

\section{Discussion:}

Phaeochromocytomas account for $90 \%$ of chromaffin tumors and about $10 \%$ originate from extra-adrenal chromaffin tissue ${ }^{6}$. Symptoms and signs of a phaeochomocytoma result from an uncontrolled release of catecholamines (norepinephrine, epinephrine, and dopamine). Once there is a suspicion, localization of tumour is usually done by CT scan, MRI or MIBG scan and the best confirmatory biochemical test is to measure 24 hours urinary $\mathrm{VMA}^{4,7}$. Surgical resection of the tumour is the only curative procedure.

Prior to surgery, it is necessary to adequately control arterial pressure, heart rate, arrhythmias and restore the blood volume to normal. Haemodynamic swings may occur at induction of anaesthesia, intubation, and during tumour handling ${ }^{4}$. Preoperative preparation is conventionally done with alpha blockers followed by beta blockers, as alpha blockade results in vasodilatation and tachycardia which is controlled by beta blockers ${ }^{4}$. Alpha blockade is commonly achieved with oral phenoxybenzamine which also permits spontaneous volume expansion but this occurs gradually and may take two to three weeks. Beta blockade should be started once alpha adrenergic blockade is fully achieved as unopposed alpha stimulation may lead to severe hypertension?. Patients usually develop hypertensive swings during surgical manipulation of the tumour despite complete pharmacological blockade. Therefore alpha and beta blockers should be continued until the day of the surgery except phenoxybenzamine which is stopped a day before surgery as it has a long half life and may cause postoperative hypotension. We preferred isoflurane as inhalational agent as it does not sensitize the myocardium to catecholamines ${ }^{10}$. Vecuronium, rocuronium and cisatracurium cause the least histamine release and have shown cardiovascular stability, so we have used vecuronium as muscle relaxant. Besides $\alpha$ and $\beta$ blockade pre-operatively, increasing anaesthetic depth plays an important role in the prevention of hypertensive crises $^{10}$. Therefore, the use of suitable anaesthetic technique to prevent hypertensive attacks is mandatory. Combination of epidural analgesia with general analgesia is not very commonly practiced for phaeochromocytoma surgery. The technique we reported in this case has the advantage over usual methods of blood pressure control in that high and extensive sympathetic blockade with epidural anaesthesia (EA) may enhance the efficacy of both, $\alpha$ blockers and $\beta$ blockers, and it might inhibit the release of catecholamines from the phaeochromocytoma and also cause vasodilatation. As in this case, the extended epidural sympathetic blockade facilitated the effectiveness of general anaesthesia and also prevented the adverse cardiovascular responses to surgical stimuli and tumor manipulations (see Figure 1).The combined technique seemed to have modified the anaesthetic course considerably, resulting in the use of fewer drugs and a steady haemodynamic state.

The three most important complications in the immediate postoperative period are hypertension, hypotension and hypoglycaemia. Nearly half of the patients remain hypertensive for a few days, probably due to elevated catecholamine levels which may persist for one week post operatively ${ }^{10}$. Hence, institution of antihypertensive therapy may be required for a few days, as in our case the patient was hypertensive post operatively for which nitroglycerine infusion was started in the ICU and finally switched over to oral $\beta$-blockers.

\section{Conclusion:}

The anaesthetic management of patients with phaeochromocytoma still remains a challenge as the pathophysiology of the disease is complex 
and a team approach of an experienced endocrinologist, endocrine surgeon and anaesthesiologist is necessary for its successful outcome. In our experience we found combined general and epidural anaesthesia to be a unique and safe technique for excision of phaeochromocytoma which could also be extrapolated for laparoscopic surgical approaches.

\section{References:}

1. Frankel Felix:Classics in oncology. A case of bilateral completely latent adrenal tumor and concurrent nephritis with changes in the circulatory system and retinitis: CA Cancer J Clin 1984; 34:93-106.

2. Webel SS, Ober P. Phaeochromocytoma: Update on diagnosis, localization and management.

3. Med Clin North Am 1995; 79: 131- 153. Saegesser F. [Cesar Roux (1857-1934) and his times]. Rev Med Suisse Romande 1984; 104:403-64.

4. Kinney MA, Narr BJ, Warner MA. Perioperative management of phaeochromocytoma. J Cardiothorac Vasc Anesth 2002; 16:359-69.

5. Prys-Roberts C. Phaeochromocytoma recent progress in its management. $\mathrm{Br} \mathrm{J}$ Anaesth 2000; 85:44-57.

6. Lenders JWM, Eisenhofer G, Manelli M, Pacak K. Phaeochromocytoma. Lancet 2005; 366:665-75.

7. Singh G, Kam P. An overview of anaesthetic issues in phaeochromocytoma. Ann Acad Med Singapore 1998; 27:843-8.

8. Bravo EL. Phaeochromocytoma. Curr Ther Endocrinol Metab 1997; 6:195-97.

9. Desmonts JM, Marty J. Anaesthetic management of patients with Phaeochromocytoma. Br J Anaesth 1984; 56:781-9.

$* * * * * * * * * * * * * * * * * * * * * * * * * * * * * * * * * * * * * * * * * * * * * * * * * * * * * * * * * * * * * * * * * * * * * * * *$

\section{THE COLLEGE OF ANAESTHESIOLOGISTS OF SRI LANKA}

\section{FREE PAPERS :}

The rules of the competition are:

1. Open to all medical practitioners in Anaesthesia.

2. Duration of the presentation must be no longer than 15 minutes.

3. The work in whole or in part should not have been presented or published elsewhere.

4. An abstract of not more than 200 words should be submitted before the closing date.

5. The first right of publication of the entries submitted lies with the editor of the College of Aanesthesiologists of Sri Lanka.

6. The decision of the panel of judges in all matters relating to the competition including questions relating to eligibility shall be final.

7. The prize will not be awarded if the Judges decide the presentation is not up to standard.

The closing date for the 2011 competition is $31^{\text {st }}$ October 2010. The abstract should reach the undersigned before the closing date. Any expenditure incurred in the preparation of the slides for papers that were selected for presentation will be reimbursed by the College. 3 copies of the full text should be submitted on or before $31^{\text {st }}$ October 2010 to the anaesthetic office. 